\title{
Searches for Gauge-Mediated SUSY Breaking Topologies with the L3 Detector at LEP
}

\author{
M. Gataullin*, S. Rosier ${ }^{\dagger}$, L. Xia ${ }^{* *}$ and H. Yang* \\ "Department of Physics, California Institute of Technology, MC 256-48, Pasadena, CA 91125, USA \\ ${ }^{\dagger}$ LAPP Annecy-le-Vieux, Chemin du Bellevue, BP 110 F-74941, CEDEX France \\ ${ }^{* *}$ Argonne National Laboratory, Argonne, IL 60439, USA \\ ${ }^{\ddagger}$ Department of Physics, University of Michigam, Ann Arbor, MI 48109, USA
}

\begin{abstract}
Searches for topologies predicted by gauge-mediated SUSY breaking models were performed using data collected with the L3 detector at LEP. All possible lifetimes of the next-to-lightest SUSY particle (NLSP), neutralino or scalar tau, were considered. No evidence for these new phenomena was found and limits on the production cross sections and sparticle masses were derived. A scan over the parameters of the minimal GMSB model was performed, leading to lower limits of $62.2 \mathrm{GeV}, 11 \mathrm{TeV}$, and $0.07 \mathrm{eV}$ on the NLSP mass, the mass scale parameter $\Lambda$, and the gravitino mass, respectively. The status of the LEP combined searches is also discussed.
\end{abstract}

Keywords: GMSB, Supersymmetry, LEP, long-lived, neutralino, scalar lepton

PACS: $12.38 . \mathrm{Qk}, 12.60 . \mathrm{Jv}$

\section{INTRODUCTION}

In this paper, we briefly review results of the searches for manifestations of the gaugemediate Supersymmetry breaking models (GMSB) in $\mathrm{e}^{+} \mathrm{e}^{-}$collisions at LEP. In GMSB, the lightest SUSY particle is always the gravitino, with a mass in the range of $10^{-2}-$ $10^{4} \mathrm{eV}$, whereas the next-to-lightest SUSY particle (NLSP) is typically the scalar tau or the lightest neutralino. The minimal GMSB model can be described by a set of the following parameters: $m_{\tilde{\mathrm{G}}}$, the gravitino mass; $\Lambda$, the universal mass scale of SUSY particles; $M_{m}$, the messenger mass; $N_{m}$, the messenger index; $\tan \beta$, the ratio between the vacuum expectation values of the two Higgs doublets; and sign $(\mu)$, the Higgs mixing parameter. Because the lifetime of the NLSP depends on the gravitino mass, NLSP decays both inside and outside the detector volume had to be considered.

Data collected by the L3 detector at LEP in the years from 1998 through 2000 were used. They correspond to an integrated luminosity of $619 \mathrm{pb}^{-1}$ at center-of-mass energies $\sqrt{s}=189-209 \mathrm{GeV}$. All results given below are considered to be preliminary. All limits are derived at the $95 \%$ confidence level.

\section{THE $\tilde{\chi}_{1}^{0}$ NLSP SCENARIO}

In the case of the neutralino NLSP, the GMSB signature is the pair-production of neutralinos, each decaying into a photon and a gravitino: $\mathrm{e}^{+} \mathrm{e}^{-} \rightarrow \tilde{\chi}_{1}^{0} \tilde{\chi}_{1}^{0} \rightarrow \tilde{\mathrm{G}} \gamma \tilde{\mathrm{G}} \gamma$.

If both neutralinos decay promptly, the experimental signature of this process is very clean, involving events with two energetic acoplanar photons. Searches for this signature 


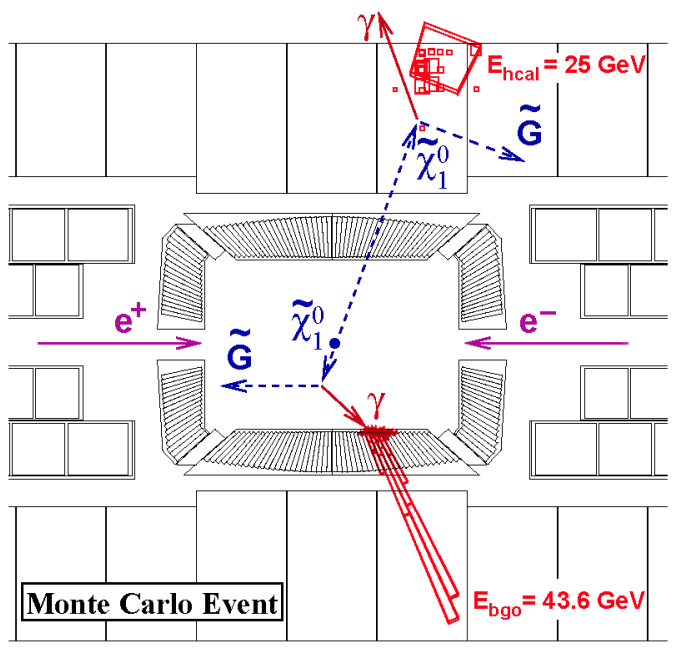

FIGURE 1. A simulated $\mathrm{e}^{+} \mathrm{e}^{-} \rightarrow \tilde{\chi}_{1}^{0} \tilde{\chi}_{1}^{0} \rightarrow \tilde{G} \tilde{G} \gamma \gamma$ event with two non-pointing photons, where $m_{\tilde{\chi}_{1}^{0}}=$ $95 \mathrm{GeV}$ and $\sqrt{s}=207 \mathrm{GeV}$. In this event one of the neutralinos decayed in front of the electromagnetic calorimeter, while the other decayed and produced a shower inside the hadron calorimeter.

were motivated by the rare ee $\gamma \gamma$ event observed by the CDF experiment. No evidence for anomalous production of such events was found and the GMSB interpretation of the CDF event was excluded [1].

However, for $m_{\tilde{\mathrm{G}}} \sim 100 \mathrm{eV}$, the proper decay length of the neutralino, $c \tau_{\tilde{\chi}_{1}^{0}}$, can become comparable to or even larger than the size of the L3 detector. In this scenario, one or both of the produced neutralinos may decay within the sensitive volume of the detector, but at a distance from the primary vertex. This would lead to events with nonpointing photons, such as shown in Figure 1.

To improve the sensitivity of this search, non-pointing photons were identified using not only the electromagnetic (ECAL) but also the hadron calorimeter (HCAL) of L3. In the ECAL, such photons were selected by studying the transverse profile of electromagnetic showers. For the signatures with neutralino decays in the HCAL, the Standard Model background was absent. However, the HCAL response to electromagnetic showers had to be measured in situ at LEP. A dedicated study was performed using events from the Bhabha scattering process, $\mathrm{e}^{+} \mathrm{e}^{-} \rightarrow \mathrm{e}^{+} \mathrm{e}^{-}$, in which both scattered electrons passed through the gaps between the ECAL barrel and endcaps and produced two showers in the HCAL. In addition, special veto cuts were applied to reject cosmic ray backgrounds.

No events with non-pointing photons were found and limits on the $\mathrm{e}^{+} \mathrm{e}^{-} \rightarrow \tilde{\chi}_{1}^{0} \tilde{\chi}_{1}^{0}$ production cross section were derived (see Figure 2a). These limits were then translated into an excluded region in the $\left(c \tau_{\tilde{\chi}_{1}^{0}}, m_{\tilde{\chi}_{1}^{0}}\right)$ plane. As shown in Figure $2 \mathbf{b}$, neutralino masses $m_{\tilde{\chi}_{1}^{0}}<88 \mathrm{GeV}$ were excluded for $c \tau_{\tilde{\chi}_{1}^{0}}$ values smaller than $100 \mathrm{~m}$. A more detailed description of this search can be found in Reference [2]. 

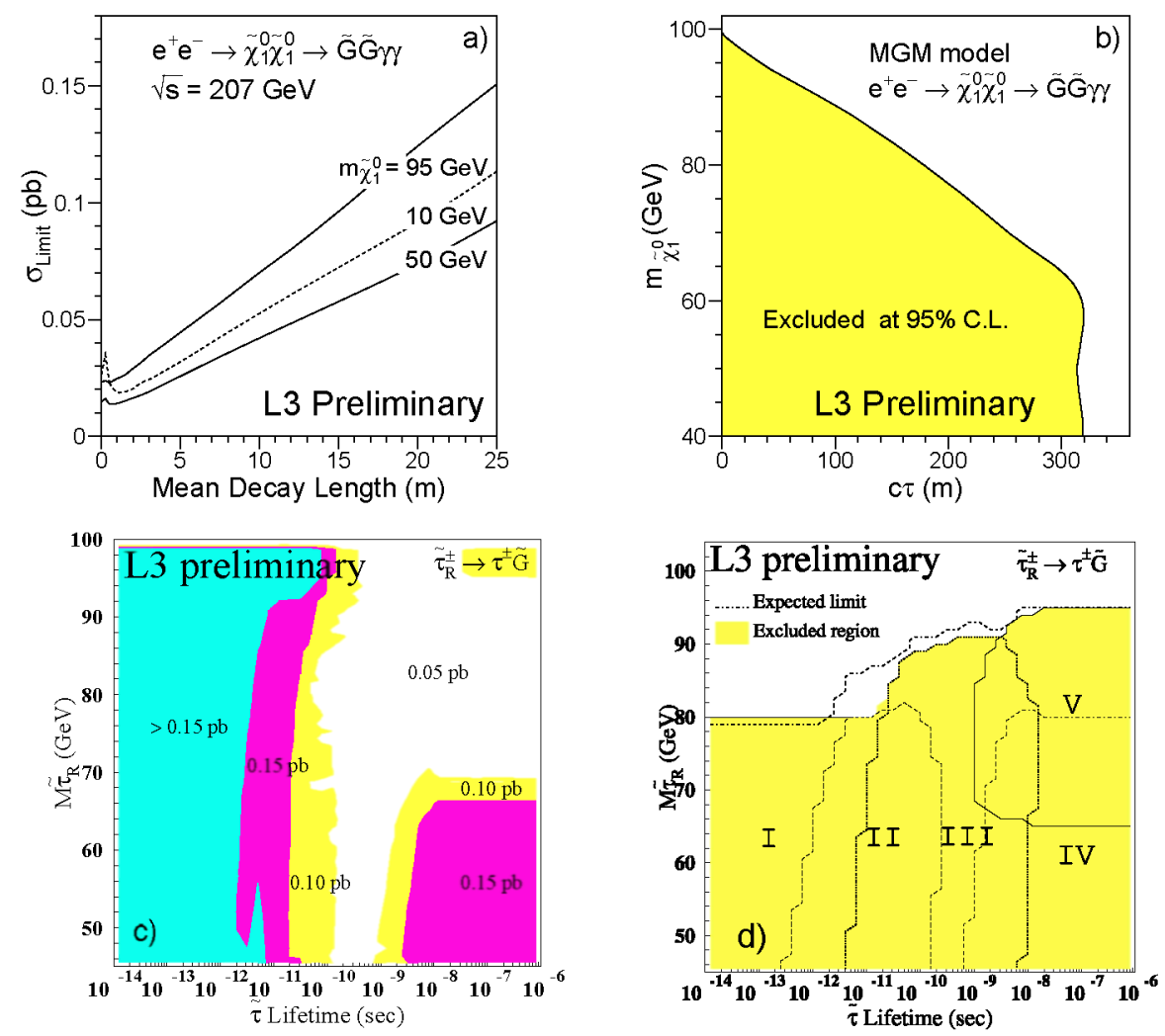

FIGURE 2. a) Upper limits on the $\mathrm{e}^{+} \mathrm{e}^{-} \rightarrow \tilde{\chi}_{1}^{0} \tilde{\chi}_{1}^{0}$ production cross section for three different neutralino mass hypotheses $m_{\tilde{\chi}_{1}^{0}}=95,50$, and $10 \mathrm{GeV}$, as functions of the mean $\tilde{\chi}_{1}^{0}$ decay length in the laboratory frame: $L_{\tilde{\chi}_{1}^{0}}=\gamma \beta c \tau_{\tilde{\chi}_{1}^{0}}$. b) Region excluded in the $\left(c \tau_{\tilde{\chi}_{1}^{0}}, m_{\tilde{\chi}_{1}^{0}}\right)$ plane under the assumptions of the MGM model [3]. c) Upper limit on the $\mathrm{e}^{+} \mathrm{e}^{-} \rightarrow \tilde{\tau} \tilde{\tau}$ production cross section at $\sqrt{s}=205 \mathrm{GeV}$, as a function of the $\tilde{\tau}$ mass and lifetime. d) Region excluded in the $\left(\tau_{\tilde{\tau}}, m_{\tilde{\tau}}\right)$ plane using five sets of different selection cuts: I prompt decay; II and III intermediate decay lengths; IV and V long-lived $\tilde{\tau}$.

\section{THE $\tilde{\tau}$ NLSP SCENARIO}

In the case of the scalar tau NLSP, the pair production process $\mathrm{e}^{+} \mathrm{e}^{-} \rightarrow \tilde{\tau} \tilde{\tau} \rightarrow \tau \tilde{\mathrm{G}} \tau \tilde{\mathrm{G}}$ was expected to be the main experimental signature. Since the corresponding final state topology depended strongly on the scalar tau lifetime, $\tau_{\tilde{\tau}}$, five different event selections were developed.

The search for acoplanar leptons covered the case of prompt $\tilde{\tau}$ decays. For this signature, the selection cuts were the same as in the search for $\tilde{\tau}$ production in gravity mediated Supersymmetry [4]. In the case of intermediate decay lengths, a search for charged tracks with large impact parameters and kinks was performed. Finally, longlived scalar taus were searched for by measuring the $\mathrm{dE} / \mathrm{dx}$ parameter of charged tracks. 
TABLE 1. Lower limits on the parameters of the minimal GMSB and sparticle masses for all possible NLSP scenarios.

\begin{tabular}{llllll}
\hline Messenger Index & $\mathbf{1}$ & $\mathbf{2}$ & $\mathbf{3}$ & $\mathbf{4}$ & $\mathbf{5}$ \\
\hline$\Lambda(\mathrm{TeV})$ & 47.3 & 25.5 & 16.8 & 13.0 & 11.0 \\
$M_{\text {mess }}(\mathrm{TeV})$ & 56 & 32 & 24 & 24 & 18 \\
$m_{\tilde{\mathrm{G}}}(\mathrm{eV})$ & 0.63 & 0.19 & 0.11 & 0.10 & 0.07 \\
$m_{\tilde{\chi}_{1}^{0}}(\mathrm{GeV})$ & 62.2 & 65.9 & 65.6 & 65.2 & 72.2 \\
$m_{\tilde{\tau}_{1}}(\mathrm{GeV})$ & 62.3 & 62.2 & 62.3 & 62.7 & 62.8 \\
$m_{\tilde{\mathrm{e}}_{\mathrm{R}}}, m_{\tilde{\mu}_{\mathrm{R}}}(\mathrm{GeV})$ & 89.6 & 73.6 & 71.2 & 66.9 & 67.1 \\
\hline
\end{tabular}

The obtained limits on the $\mathrm{e}^{+} \mathrm{e}^{-} \rightarrow \tilde{\tau} \tilde{\tau}$ cross section are shown in Figure $2 \mathrm{c}$. These limits were then translated into a limit on the $\tilde{\tau}$ mass: $m_{\tilde{\tau}}>80 \mathrm{GeV}$ (see Figure $2 \mathrm{~d}$ ). A detailed description of this search can be found in Reference [5].

\section{COMBINATIONS OF SEARCH RESULTS}

To combine results of the searches performed for all possible NLSP scenarios, including those not described above, a scan over the GMSB parameter space was performed [6]. In total, 5.6 $\cdot 10^{6}$ points in the parameter space were tested. At each point the complete mass spectrum, production cross sections, and branching ratios were calculated. A point in the parameter space was considered excluded if it was kinematically accessible and the calculated cross section was higher than the cross section limit for the corresponding discovery channel. The results of this scan are summarized in Table 1.

For the $\tilde{\tau}$ NLSP scenario, a combination of the searches by all four LEP experiments was performed, excluding $\tilde{\tau}$ masses below $86.9 \mathrm{GeV}$ [7]. A LEP-wide combination also exists for the short-lived $\tilde{\chi}_{1}^{0}$ NLSP scenario [8].

\section{ACKNOWLEDGMENTS}

We would like to thank all our colleagues from the L3 collaboration. This work was supported in part by the U.S. Department of Energy Grant No. DE-FG03-92-ER40701.

\section{REFERENCES}

1. P. Achard et al. [L3 Collaboration], Phys. Lett. B 587, 16 (2004).

2. M. Gataullin, Studies of Electroweak Interactions and Searches for New Physics Using Photonic Events with Missing Energy at the Large Electron-Positron Collider, Ph.D. thesis, Caltech, 2006.

3. S. Dimopoulos, S. Thomas, and J.D. Wells, Phys. Rev. D 54, 3283 (1996).

4. P. Achard et al. [L3 Collaboration], Phys. Lett. B 580, 37 (2004).

5. L. Xia, Search for Scalar Leptons at LEP with the L3 Detector, Ph.D. thesis, Caltech, 2003.

6. M. Gataullin, S. Rosier-Lees, L. Xia, and H. Yang, L3 Note 2777, October 2003.

7. LEP SUSY Working Group, note LEPSUSYWG/02-09.2, http://epsusy.web.cern.ch/

8. LEP SUSY Working Group, note LEPSUSYWG/04-09.1, http://lepsusy.web.cern.ch/ 